\title{
Rainbow on a Chip: Experimental Observation of the Trapped Rainbow Effect Using Tapered Hollow Bragg Waveguides
}

\author{
Aaron Melnyk \\ Faculty of Engineering, University of Alberta
}

\begin{abstract}
Experimental observation of the 'trapped rainbow' in the visible is demonstrated using tapered hollow Bragg waveguides. These waveguides spatially disperse an input spectrum into its various frequency components and vertical out of plane radiation was observed at wavelength dependant positions along the entire length of the waveguide. The experimental observation is corroborated by a brief theoretical analysis and simulation. These devices form the foundation for future work involving integration into a micro-spectrometer for eventual lab-on-chip use.
\end{abstract}

\section{OVERVIEW AND MOTIVATION}

Slow light phenomena and light trapping has been an area of interest for many years. In 2007 Tsakmakidis et al. coined the term 'trapped rainbow' for the slowing and spatial dispersion of white light in metamaterial structures [1]. This unique effect has led many to propose its use in a wide variety of optoelectronic devices including photonic logic circuits [2], communication networks [3], signal processors [3] and optical data processing [1]. While in principle the trapped rainbow phenomena can be realized, currently no such material exists that can stop and trap light indefinitely in this manner over a broad range of wavelengths [2]. Nonetheless there have been a few reports of experimental observation of the effect, notably Qiaoqiang Gan and Filbert J. Bartoli [2] and V.N. Smolyaninova et al. [4]. The former utilized chirped nanogratings while the latter emulates the necessary metamaterial properties using coated convex lenses. This paper, however, focuses on the application of the 'trapped rainbow' for on-chip spectroscopy, where the spatial separation and stopping of an input spectrum is extremely attractive.

In regards to this proposition, the global spectrometer market is expected to reach a value of $\$ 4$ billion this year [5]. This is in parallel with the lab-on-chip (LOC) market which is expected to reach a value of $\$ 3$ billion in the United States this year with an annual growth rate of $25 \%$ [6]. LOCs often employ the use of fluorescent signals to indicate the presence or absence of biomarkers of interest [7], making a micro-spectrometer a perfect sensing device for these diagnostics tools. The burgeoning spectrometer and analytical biochip markets present a unique research opportunity, where two industries are experiencing simultaneous growth while at the same time still require considerable amounts of development and innovation.

While prior results have been successful to varying degrees, they do not provide optimal geometries or properties for use in a on-chip spectroscopy. However, prior research by Dr. DeCroby's group in tapered waveguides using omnidirectional dielectric reflectors (ODRs) in the near IR region has offered promising results for microspectrometer use [8]. Here the ODRs confine in-coupled light toward the narrow end of the taper.
Wavelength dependant cut-off positions exists causing the in-coupled spectrum to be spatially disperesed and out of plane radiation to occur. This externally radiated light can then be coupled to a photo detector array for use as a spectrometer. It is the focus of this paper to transfer this design to the visible light region and realize the 'trapped rainbow' effect.

\section{THEORY AND DESIGN}

The proposed spectrometer relies on ODRs which have the advantage over metallic mirrors of being low loss while still achieving extremely high reflectance $[9,10]$. These attributes allow guided light to propagate with minimal energy loss down the tapered waveguide to wavelength dependant cut-off positions. The structure of ORDs consist of alternating high and low index of refraction materials tuned to a specific wavelength, the Bragg wavelength $\left(\lambda_{B R A G G}\right)$, defined as:

$$
\lambda_{B R A G G}=4 n_{1} d_{1}=4 n_{2} d_{2}
$$

Where $n_{1}$ and $n_{2}$ are the indices refraction and $d_{1}$ and $d_{1}$ are the thicknesses of alternating layers 1 and 2 respectively. This equation defines the quarter-wave stack (QWS) condition; light centered around $\lambda_{B R A G G}$ experience high reflectance due to constructive interference of the partially reflected rays as they propagate through each layer. Reflectance of these structures can be optimized by increasing the number of layers, using an odd number of layers, and when the high-index material is outermost [9].

Unfortunately dielectric mirrors only offer high reflectance at all angles for a small range of wavelengths; the omnidirectional band. This band can be maximized by increasing the index contrast between the alternating layers in the QWS structure [11, 12] (Fink et al.[11] presents an in depth theoretical analysis of this condition). Moreover, by setting the index of the low index material around 1.45, the omnidirectional band can be maximized [13].

Based on prior familiarity and ease of use with available e-gun deposition systems, $\mathrm{TiO}_{2} / \mathrm{SiO}_{2}$ were chosen for use in our devices. Setting $\lambda_{B R A G G}$ to $550 \mathrm{~nm}$, 


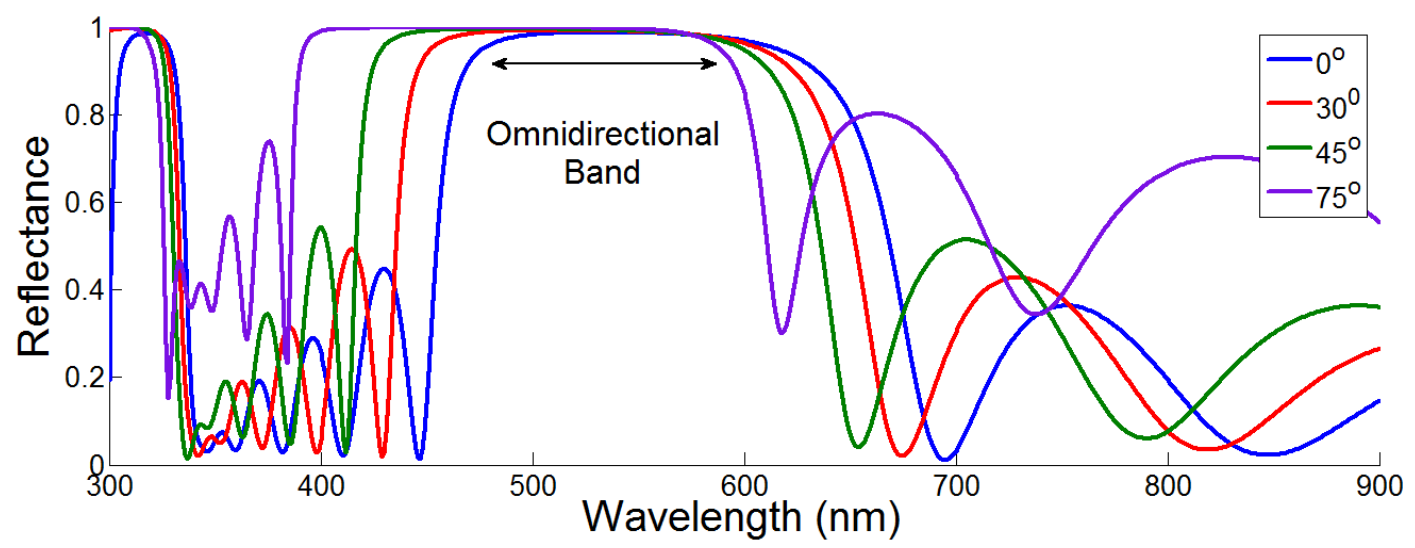

FIG. 1. Modeled reflectance of a 6.5 period $\mathrm{TiO}_{2} / \mathrm{SiO}_{2}$ Bragg mirror at various angles. The omnidirectional band spans wavelenghts $\sim 490 \mathrm{~nm}-570 \mathrm{~nm}$.

thicknesses of $\mathrm{TiO}_{2}(n \sim 2.35)$ and $\mathrm{SiO}_{2}(n \sim 1.48)$ were determined to be $61 \mathrm{~nm}$ and $93 \mathrm{~nm}$ respectively.

MATLAB code was used to simulate properties of the ODRs and then the tapered waveguide structures using the standard transfer matrix model [14], which is based on the electromagnetic continuity equations across boundaries. This method when applied to a QWS structure will produce a system matrix which provides a mathematical description of the light as is passes through the entire structure. An in depth analysis of this method as used in tapered waveguides with ODR claddings can be found elsewhere $[15,16]$.

Taking into account device mass, deposition time, and cost, 6.5 period (13 layer) Bragg reflectors were selected and modeled with $\mathrm{TiO}_{2}$ as the terminating layer using the thicknesses derived above. Figure 1 displays the reflectance of these mirrors at a variety of angles with the omnidirectional band presented. The reflectance in the band exceeds $98 \%$, with the band itself spanning $80 \mathrm{~nm}$ (from $\sim 490-570 \mathrm{~nm}$ ).

Figure 2 considers a ray-optics representation of a tapered waveguide clad with ODRs described above. A

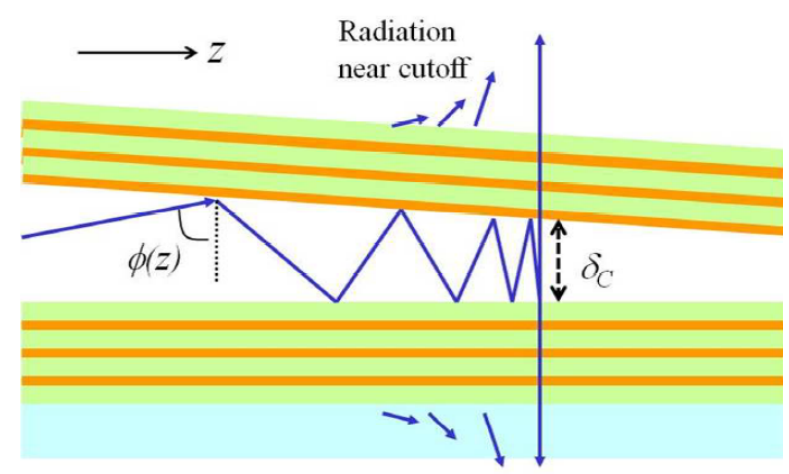

FIG. 2. Ray diagram of a tapered waveguide clad with omnidirectional reflectors [8]. given mode of light will propagate through the waveguide core until it reaches a specific cut-off thickness, $\delta_{c n}$. This cut-off position is defined as the core thickness of the waveguide that realizes a Fabry-Perot cavity [15] for that specific wavelength and mode. The general equation for cut-off the $n t h$ order mode is thus:

$$
\delta_{c n}=(n+1) \lambda_{o} / 2
$$

With $\lambda_{o}$ the free space wavelength and mode ordering $n=0,1,2, \ldots$. At this cut-off position the "trapped" light will begin to radiate vertically outward. A microspectrometer can be realized by coupling a photodetector array the cut-off location of light of interest. Such a device holds key advantages over other microspectrometers, namely: scalability, the lack of complex moving parts, and a high potential throughput due to the low loss and dispersive nature of the waveguide.

\section{FABRICATION}

Electron beam evaporation was used to deposit the $\mathrm{TiO}_{2} / \mathrm{SiO}_{2}$ layers on separate silicon and glass substrates. $\mathrm{SiO}_{2}$ was deposited at a pressure of $1.5 * 10^{-6}$ Torr at a deposition rate of $\sim 3 \AA / s$. $\mathrm{TiO}_{2}$ was deposited with a partial pressure of oxygen (to achieve the required stoichiometry) at a pressure of $5 * 10^{-5}$ Torr and a deposition rate of $0.5 \AA / \mathrm{s}$. The optical properties of the deposited mirrors were then verified using variable angle spectroscopic ellipsometry (VASE) and profilometer measurements and were found to be in close accordance with the simulation for a wide variety of angles, confirming the omnidirectionality criteria.

The mirrors were then fabricated into tapered waveguides, the final result of which is illustrated in Fig 3(a). First the glass and silicon substrates were cleaved and diced into small dimensions in order to create waveguides with lengths of $4 \mathrm{~mm}$. Drops of a UV curable 


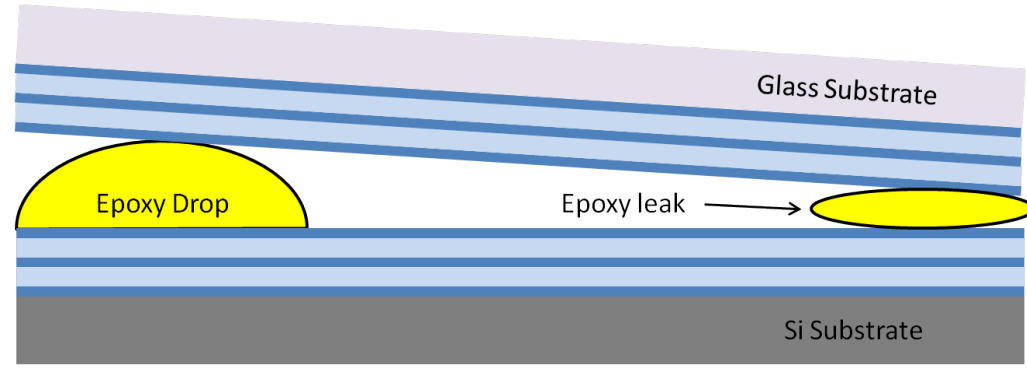

(a)

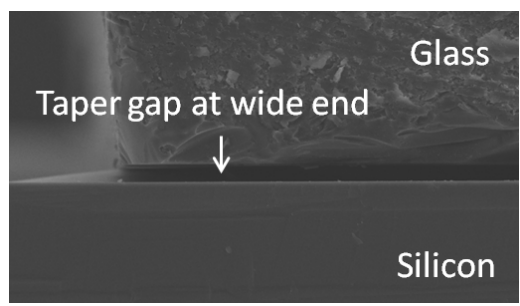

(b)

FIG. 3. (a) Illustrative side view of the fabricated waveguide with the epoxy leak visible. (b) SEM image taken of the device at the wide end of the taper.

epoxy (NOA 61, Norland) were next deposited and cured along one side of the cleaved pieces of silicon to act as permanent 'posts'. A diced piece of the glass substrate was then aligned on top of the silicon substrate (deposited layers facing one another) where the epoxy 'posts' prop up the glass at one end. In this way the silicon substrate acts as the bottom waveguide cladding while the glass substrate provides the top cladding. The peices were then bonded together at the narrow end using the NOA 61. The taper slope is thus defined at the height of the epoxy 'posts' divided by the length of the waveguide $(4 \mathrm{~mm})$.

The periodically deposited drops of epoxy (positioned $\sim 200 \mu \mathrm{m}$ apart) were measured with a profilometer and produced relatively uniform feature heights, ranging from $11-13 \mu \mathrm{m}$. The taper profile of the compiled waveguides were then confirmed using SEM images (see Fig. 3(b)). SEM analysis also revealed that the substrates did not come into complete contact at the rear, instead epoxy had leaked between the substrates prohibiting contact. This had the effect of propping up the rear at a height of $2 \mu \mathrm{m}$. This grievance is illustrated in Fig. 3(a).

For experimental purposes the leaked epoxy has the effect of preventing roughly the first 6-7 modes from reaching cut-off since their cut-off positions appear at core heights less than $2 \mu \mathrm{m}$. Given this and the known post height we can estimate the number of supported modes for light of $594 \mathrm{~nm}$ and $532 \mathrm{~nm}$ to be $\sim 37$ and $\sim 41$ respectively with subsequent mode spacing of $0.11 \mathrm{~mm}$ and $0.098 \mathrm{~mm}$ given a waveguide length of $4 \mathrm{~mm}$.

\section{EXPERIMENTAL RESULTS}

In experiments a 532nm green laser and 594nm amber laser were free space coupled to the wide (input) end of the slab waveguide structures. A broadband white light source (Koheras SuperK Red) was also coupled to the waveguides using a multi-mode fibre. Light radiated/scattered out of the top waveguide cladding (glass substrate) was captured using a visible spectrum camera coupled to objective lenses. A schematic diagram can be

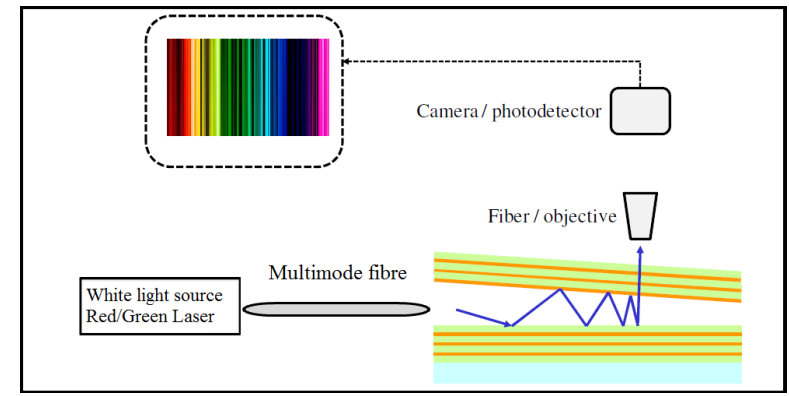

FIG. 4. Schematic view of the experimental setup.

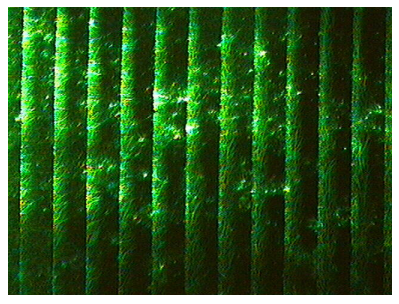

(a)

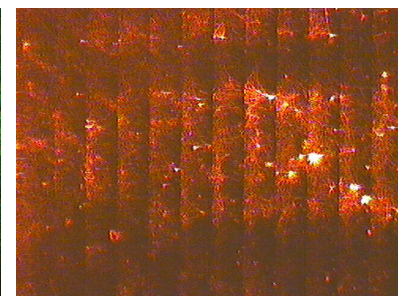

(b)
FIG. 5. Images captured of the vertically radiated light at mode cut-off for (a) the $594 \mathrm{~nm}$ laser and (b) the $532 \mathrm{~nm}$ laser. The bright bands correspond the standing wave patterns that form at cutoff.

seen in Fig. 4.

Images of radiated light from the $532 \mathrm{~nm}$ and 594nm laser are provided in Fig. 5. Periodic bands of laser light can be seen along the length of the images. Based on the previous discussions, the peak intensity lines are attributed to the various modes of the input laser source forming standing waves defined by the Fabry-Perot resonance condition, where the mode is 'trapped' and forward propagation halted. While only a portion of the waveguide is imaged here, these fringes were present along the entire $4 \mathrm{~mm}$ length of the waveguides. Bands from the green laser appear sharper and more defined when compared to the amber laser which can be attributed to the proximity of $594 \mathrm{~nm}$ light to the upper limit of the designed PBG which in turn negatively in- 
fluences confinement and reflection in the waveguide. In total, depending on the individual sample tested, the number of modes present along the length of the waveguide numbered 39-43 and 36-39 for 532nm and 594nm light respectively, comparing quite well to the estimated number of supported modes within the waveguides.

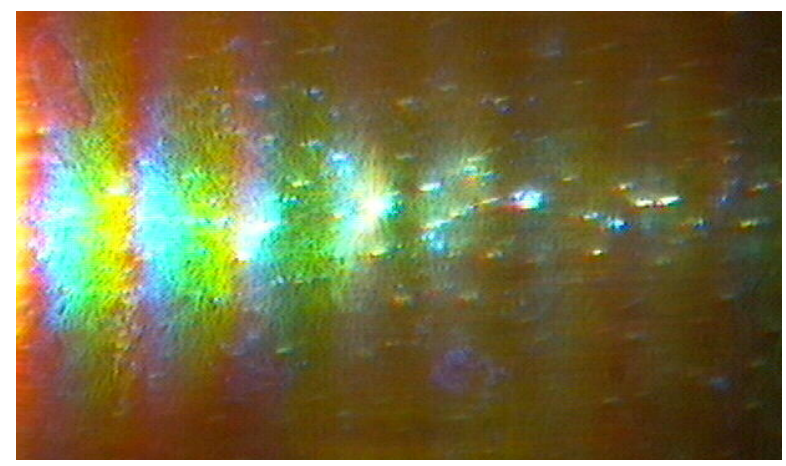

FIG. 6. 'Trapped rainbow' from broadband white light illumination. Colours visibly undergoing cut-off include blue, cyan, green and yellow indicating a wavelength range of $\lambda \sim$ 450nm-590nm.

Lastly the Koheras white light source was coupled to the waveguide input using a multi-mode fibre to verify the 'trapped rainbow', or mode cut-off all light within the PBG of the devices. An image of vertically radiated light from this source is presented in Fig. 6; the spatial dispersion and 'trapped rainbow' effect of the input spectrum visible. Each supported mode experiences cut-off with several colours visibly seen radiating vertically outward including blue, cyan, green and yellow. This equates to a wavelength range of $\lambda \sim 450 \mathrm{~nm}-$ $590 \mathrm{~nm}$, which is in reasonable accordance to the simulated PBG of the reflectors as presented in Fig 1.

\section{CONCLUSION AND FUTURE WORK}

To conclude, 'rainbow trapping' in the range $\lambda=$ $450 \mathrm{~nm}-590 \mathrm{~nm}$ was experimentally demonstrated using a tapered waveguide structure clad with omnidirectional dielectric reflectors and illuminated with $532 \mathrm{~nm} / 594 \mathrm{~nm}$ lasers and a broadband white light source. Comparing results to other experimental observations of the 'trapped rainbow' [2,4], these waveguides have the potential to offer lower loss and thus longer light "storage" characteristics due to the all dielectric structure. In respect to the LOC integration, modifying the fabrication techniques to provide an enclosed hollow waveguide core would allow for the cavity to potentially be filled with a liquid or gas analyte [7]. Potential fabrication methods to achieve this modified structure include a monolithic assembly using induced buckling [17-19] or grey-scale lithography and sacrificial etching [8]. These methods would also provide the necessary methods to eliminate the 'epoxy leakage' issue since they do not depend on such bonding techniques.

Looking forward, we wish to amend the fabrication techniques and employ such a waveguide directly into the proposed mircospectrometer by integrating photodetector arrays. This will allow direct comparison to other microspectrometers being developed such as tunable MEMS devices [20] or filter array and wedge filter spectrometers [21, 22].

\section{ACKNOWLEDGMENTS}

I would like to thank Dr. Ray DeCorby for his role as my mentor for this project, always insightful and willing to help. I would also like to awknowledge Trevor Allen, Brian Drobot and Minchang Zhang for their assistance in fabrication and experimental testing.

\section{REFERENCES}

[1] Kosmas L. Tsakmakidis, Allan D. Boardman \& Ortwin Hess. Trapped rainbow storage of light in metamaterials. Nature 450, 397-401 (15 November 2007).

[2] Qiaoqiang Gan and Filbert J. Bartoli. Surface dispersion engineering of planar plasmonic chirped grating or complete visible rainbow trapping. Appl. Phys. Lett. 98, 251103 (2011)

[3] X. P. Zhao, W. Luo, J. X. Huang, Q. H. Fu, K. Song et al. Trapped rainbow effect in visible left-handed heterostructures. Appl. Phys. Lett. 95, 071111 (2009).

[4] Vera N. Smolyaninova, Igor I. Smolyaninov, Alexander V. Kildishev, and Vladimir M. Shalaev. Experimental observation of the trapped rainbow. Appl. Phys. Lett. 96211121 (2010).

[5] Dr. J. Charles Forman. Spectroscopy: Market Research Report. BCC Research, 2010

[6] Steven Hener. Analytical Chip Technology Markets. Kalorama Information, 3rd Ed., 2007.

[7] O. Schmidt, M. Bassler, P. Kiesel, C. Knollenberg, and N. Johnson, Fluorescence spectrometer-ona-fluidicchip, Lab Chip 7(5), 626629 (2007).

[8] R.G. DeCorby, N. Ponnampalam, E. Epp, T. Allen, J.N. McMullin. Chip-scale spectrometry based on tapered hollow Bragg waveguides. Optics Express, vol. 17 no.19, pp 16632-16645, Sept. 142009.

[9] H. A. Macleod, Thin-Film Optical Filters, Taylor \& Fran- cis, 2001

[10] S.O. Kasap, Optoelectronics and Photonics: Principles and Practices, Prentice Hall, 2001

[11] Y. Fink, J. N. Winn, F. Shanhui, C. Chiping, J. Michel, J. D. Joannopoulos, and E. L. Thomas, A dielectric omnidirectional reflector, Science 282, 1679-1682 (1998).

[12] J. P. Dowling, Mirror on the Wall: Youre Omnidirectional After All, Science 282, 1841 (1998). 
[13] W. H. Southwell, Omnidirectional mirror design with quarterwave dielectric stacks, Appl. Opt. 38, 54645467 (1999).

[14] E. Hecht, Optics, 4th ed. Addison Wesley, 2001.

[15] N. Ponnampalam, and R. G. DeCorby, Out-of-plane coupling at mode cutoff in tapered hollow waveguides with omnidirectional reflector claddings, Opt. Express 16(5), 28942908 (2008).

[16] N. Ponnampalam, R.G. DeCorby. Analysis and fabrica- tion of hybrid metal-dielectric omnidirectional Bragg reflectors. Applied Optics, vol. 47 no. 1pp 3037, Dec. 202007

[17] N. Ponnampalam, and R. G. Decorby, Selfassembled hollow waveguides with hybrid metaldielectric Bragg claddings, Opt. Express 15(20), 1259512604 (2007).

[18] Epp, E.; Ponnampalam, N.; Newman, W.; Drobot, B.; McFarlane, S.; McMullin, J.N.; Meldrum, A.; DeCorby, R.G.; Silicon-based integrated hollow waveguides produced by controlled thin film buckling, IEEE Photonics Society, 2010 23rd Annual Meeting of the , vol., no., pp.258-259, 7-11 Nov. 2010.

[19] R. G. DeCorby, N. Ponnampalam, H. T. Nguyen, M. M. Pai, and T. J. Clement, Guided self-assembly of integrated hollow Bragg waveguides, Opt. Express 15, 3902- 3915 (2007).

[20] R. A. Crocombe, D. C. Flanders, and W. Atia, Microoptical instrumentation for process spectroscopy, Proc. SPIE 5591, 1125 (2004)

[21] S.-W. Wang, C. Xia, X. Chen, W. Lu, M. Li, H. Wang, $\mathrm{W}$. Zheng, and T. Zhang, Concept of a high-resolution miniature spectrometer using an integrated filter array, Opt. Lett. 32(6), 632634 (2007).

[22] O. Schmidt, P. Kiesel, and M. Bassler, Performance of chip-size wavelength detectors, Opt. Express 15(15), 97019706 (2007). 